University of Wollongong

Research Online

Australian Institute for Innovative Materials -

Papers

Australian Institute for Innovative Materials

$1-1-2014$

Performance enhancement of single-walled nanotube-microwave exfoliated graphene oxide composite electrodes using a stacked electrode configuration

\author{
Dennis Antiohos \\ University of Wollongong, dennisa@uow.edu.au \\ Mark S. Romano \\ University of Wollongong, mromano@uow.edu.au \\ Joselito M. Razal \\ University of Wollongong, jrazal@uow.edu.au \\ Stephen Beirne \\ University of Wollongong, sbeirne@uow.edu.au \\ Phil Aitchison \\ Cap-Xx (Australia) Pty Ltd, phil.aitchison@cap-xx.com
}

See next page for additional authors

Follow this and additional works at: https://ro.uow.edu.au/aiimpapers

Part of the Engineering Commons, and the Physical Sciences and Mathematics Commons 


\title{
Performance enhancement of single-walled nanotube-microwave exfoliated graphene oxide composite electrodes using a stacked electrode configuration
}

\author{
Abstract \\ We report the development of a stacked electrode supercapacitor cell using stainless steel meshes as the \\ current collectors and optimised single walled nanotubes (SWNT)-microwave exfoliated graphene oxide \\ (mw rGO) composites as the electrode material. The introduction of mw rGO into a SWNT matrix creates \\ an intertwined porous structure that enhances the electroactive surface area and capacitive performance \\ due to the 3-D hierarchical structure that is formed. The composite structure was optimised by varying the \\ weight ratio of the SWNTs and mw rGO. The best performing ratio was the $90 \%$ SWNT- $10 \% \mathrm{mw}$ rGO \\ electrode which achieved a specific capacitance of $306 \mathrm{~F} \mathrm{~g}-1$ (3 electrode measurement calculated at 20 \\ $\mathrm{mV} \mathrm{s}-1$ ). The $90 \%$ SWNT- $10 \% \mathrm{mw}$ rGO was then fabricated into a stacked electrode configuration (SEC) \\ which significantly enhanced the electrode performance per volume (1.43 mW h cm-3, \& $6.25 \mathrm{~W} \mathrm{~cm}-3)$. \\ Device testing showed excellent switching capability up to $10 \mathrm{~A} \mathrm{g-1}$, and very good stability over 10000 \\ cycles at $1.0 \mathrm{~A}$ g-1 with $93 \%$ capacity retention.
}

\section{Keywords}

single, walled, nanotube, configuration, electrode, stacked, electrodes, exfoliated, graphene, oxide, composite, performance, enhancement, microwave

Disciplines

Engineering | Physical Sciences and Mathematics

\section{Publication Details}

Antiohos, D., Romano, M. S., Razal, J. M., Beirne, S., Aitchison, P., Minett, A. I., Wallace, G. G. \& Chen, J. (2014). Performance enhancement of single-walled nanotube-microwave exfoliated graphene oxide composite electrodes using a stacked electrode configuration. Journal of Materials Chemistry A, 2 (36), 14835-14843.

\section{Authors}

Dennis Antiohos, Mark S. Romano, Joselito M. Razal, Stephen Beirne, Phil Aitchison, Andrew I. Minett, Gordon G. Wallace, and Jun Chen 


\title{
Performance enhancement of single-walled nanotube - microwave exfoliated graphene oxide composite electrodes using a stacked electrode configuration.
}

\author{
Dennis Antiohos, Mark S. Romano, Joselito M. Razal, Stephen Beirne, Phil \\ Aitchison, Andrew I. Minett, Gordon G. Wallace*, Jun Chen*
}

\begin{abstract}
We report the development of a stacked electrode supercapacitor cell using stainless steel meshes as the current collectors and optimised single walled nanotubes (SWNT) / microwave exfoliated graphene oxide ( $\mathrm{mw}$ rGO) composites as the electrode material. The introduction of mw rGO into a SWNT matrix creates an intertwined porous structure that enhances the electroactive surface area and capacitive performance due to the 3-D hierarchical structure that is formed. The composite structure was optimised by varying the weight ratio of the SWNTs and mw rGO. The best performing ratio was the $90 \%$ SWNT $-10 \% \mathrm{mw} \mathrm{rGO}$ electrode which achieved a specific capacitance of $306 \mathrm{~F} / \mathrm{g}$ (3 electrode measurement calculated at $20 \mathrm{mV} / \mathrm{s}$ ). The $90 \% \mathrm{SWNT}-10 \% \mathrm{mw}$ rGO was then fabricated into a stacked electrode configuration (SEC) which significantly enhanced the electrode performance per volume (1.43 $\mathrm{mW} . \mathrm{hcm}^{-3}, \& 6.25 \mathrm{Wcm}^{-3}$ ). Device testing showed excellent switching capability up to $10 \mathrm{~A} / \mathrm{g}$, and very good stability over 10000 cycles at $1.0 \mathrm{~A} / \mathrm{g}$ with $93 \%$ capacity retention.
\end{abstract}

\section{Introduction}

Supercapacitors or electrochemical capacitors are an intensely researched area for energy storage and conversion due to their high power densities, high energy densities, and long life cycles [1]. Current commercial uses of supercapacitors include personal electronics, mobile communications, back-up power and storage and industrial power and energy management [2, 3]. A recent application is the use of supercapacitors in the emergency doors on the Airbus A380, highlighting their safe and reliable performance[3]. Supercapacitors can have designs including stacked and spiral wound which maximises the performance and efficiency [1].

An ideal graphene sheet is a single-atom thick nanostructured sheet arranged in a two dimensional honeycomb lattice exhibiting a theoretical surface area of $2630 \mathrm{~m}^{2} / \mathrm{g}$, giving it the potential for the use in electrochemical devices [4, 5]. Economically speaking, graphitic forms of carbon are widely available, while large scale synthesis of graphene (and its derivatives such as graphene oxide) being readily simple procedures to perform $[5,6]$. The advantage of graphene is its ability to be used as a high surface area conducting scaffold in a given electrode system, which not only provides good electrical properties but a robustness and support of the structure [6]. Its disadvantage is that the conductivity of polycrystalline graphene is limited by the high resistance of the grain boundaries [7]. CNTs are materials of also a great interest in the area of energy storage and conversion due to their favourable electrical and mechanical properties, as well as low cost and abundance [8]. The theoretical amount of current a CNT can carry is $10^{9} \mathrm{~A} / \mathrm{cm}^{2}$ along its length, while Young's modulus ranges from $1054-1200 \mathrm{GPa}[9,10]$. CNTs are thus able to enhance the conductivity of electrode systems [11].

The mechanical, chemical, and electronic properties, coupled with the high aspect ratio of graphene make it attractive for applications in composite materials [12]. Composites containing CNTs and graphene are materials of particular interest in the energy storage and conversion area due to their favourable properties, which can result in unique optical, electrical, magnetic and chemical properties substantially different from the individual components[4, 1316]. It has been shown that the combination of both CNTs and graphene allows an expressway of electron transport from the electrode material to the current collector [17]. The ability of the CNTs to wrap around the graphene sheets helps to create a high electroactive surface area (ESA) electrode while increasing electrical conductivity and mechanical stability [4, 17-19]. The combination of high surface area and nanoscopic 
charge separation can potentially lead to a material with high energy and power density $[12,19]$.

In this paper, we describe a facile method to develop electrodes of SWNT and exfoliated graphene oxide (mw rGO) with varying compositions via sonication, centrifugation and vacuum filtration. These composites are then optimised with the best performing weight ratio used in the fabrication of a supercapacitor with a stacked electrode configuration (SEC). The reason for the SEC is to increase the amount of material that can be used in a single device, while still allowing the electrolyte to interact with as much surface area as possible. In current commercial supercapacitor systems, multiple devices are configured in series and / or parallel depending on the voltage and current needs [20]. This leads to a certain space requirement, and the more devices needed the more space that is used. By employing a SEC, it is possible to reduce the amount of space needed for supercapacitor systems which is important for industrial applications where device foot print needs to be minimised [1]. Based on the properties and performance described in this paper, the composite created has potential to significantly enhance the energy density of supercapacitors systems.

\section{Experimental}

\section{Preparation of microwave exfoliated GO.}

Graphene oxide was synthesised using a modified Hummers method as outlined by Marcano et. al. [21]. In detail, $1 \mathrm{~g}$ of graphite powder (Bay Carbon Inc.) was added to $60 \mathrm{ml}$ of concentrated (98\% w/v) $\mathrm{H}_{2} \mathrm{SO}_{4}$ (Univar) and mixed thoroughly for a few minutes. Then $3.5 \mathrm{~g}$ of $\mathrm{KMNO}_{4}$ (Sigma-Aldrich) was added in small aliquots so as the temperature did not exceed $100{ }^{0} \mathrm{C}$. This mixture was left stirring overnight for 18 hours. $300 \mathrm{ml}$ to $500 \mathrm{ml}$ of distilled $\mathrm{H}_{2} \mathrm{O}$ was added (ice bath condition) to hydrolyse the intercalation compound that forms graphite oxide. Lastly, 30\% aqueous $\mathrm{H}_{2} \mathrm{O}_{2}$ (Univar) (drop wise, approximately $3 \mathrm{ml}$ ) is added until a complete colour change is observed. The $\mathrm{H}_{2} \mathrm{O}_{2}$ is added after hydrolysis to decompose permanganate ions into manganese (IV) ions where after the manganese (IV) ions are removed by vacuum filtration (PVDF membrane, 0.22 micron pore size) by washing twice with concentrated (36 \% w/v) $\mathrm{HCl}$ (Univar), water, and ethanol. The $\mathrm{HCl}$ ensures no undesirable manganese hydroxides form, which can get trapped in between the graphene / graphite layers[22]. After vacuum filtration the slurry is dried in a vacuum oven overnight at $50{ }^{0} \mathrm{C}$.

Exfoliated GO (mw rGO) was formed by using a commercial microwave-chemistry system (APEX, Shanghai EU Microwave Chemistry Technology, Co. Ltd.). After an irradiation time of approximately 5 - $10 \mathrm{sec}$ under the power of 1000W, the GO glowed red-hot accompanied by fuming and sparking, leading to a remarkable volume expansion caused by the violent expulsion of the volatile species from the interlayer spaces of the graphene intercalation compound [23, 24].

Synthesis and fabrication of SWNT- mw rGO electrodes.
SWNTs (HiPCo,Continental Carbon Nanotechnologies Inc and mw rGO were dispersed using 1-cyclohexyl-2-pyrrolidone (CHP) at a concentration of $1 \mathrm{mg} / \mathrm{ml}$ using a combination of probe sonication at 30\% amplitude for 60 minutes, (Branson probe sonifier) and bath (Branson B1500R-MT) for 90 minutes. The dispersion was left overnight for reaggregation to occur after which centrifugation (Eppendorf Centrifuge 5415 D) was carried for 90 minutes at $2500 \mathrm{rcf} / 4000 \mathrm{rpm}$. The concentration of the supernatant was determined via Ultra Violet-Visible Spectroscopy (UV-VIS) using a Shimadzu UV1800. The sample was then diluted to a concentration of 0.4 $\mathrm{mg} / \mathrm{ml}$.

SWNT-mw rGO composites were prepared at the following weight ratios: 100-0, 95-5, 90-10, 85-15, 80-20, 50-50, 40-60, and $0-100$. Dispersions of SWNT and mw rGO were mixed via a combination of probe sonication for 30 minutes at $30 \%$ amplitude (1 sec on / 1 sec off pulse), bath sonication for 90 minutes. Films having varying thicknesses of 1, 1.5, 2.5, 4.5, $6.5,10.5,17$, and 21.5 micron were made using vacuum filtration onto platinum sputter coated (100 nm thickness) Millipore PVDF membrane with a pore size of $0.1 \mathrm{um}$ and thickness of $100 \mu \mathrm{m}$. All electrodes were plasma treated for 20 minutes to enhance the wettability of the electrode in aqueous environments. The plasma treatment used a Harrick Plasma Cleaner PDC-32G-2 and Plasmaflo PDC-FMG. Air flow was kept at 1100 mTorr for 20 minutes. The composite was removed from the platinized PVDF membrane for device testing.

\section{Electrochemical characterisation.}

Electrochemical studies of the fabricated electrodes with varied weight ratios and thicknesses were performed using a three electrode set up with a Pt mesh counter $\left(2 \mathrm{~cm}^{2}\right)$, and $\mathrm{Ag} / \mathrm{AgCl}$ reference electrode in $1 \mathrm{M} \mathrm{NaNO} \mathrm{N}_{3}$ in order to determine the optimum electrode composition electrochemical response and kinetic response as a function of thickness. Upon optimization of electrode composition and thickness of 90\%SWNT-10\% mw rGO was determined, supercapacitor device testing occurred using a Swagelok system where a nylon union tube fitting was modified to house two stainless steel current collectors as can be seen by Figure 6a. This type of cell is commonly used in supercapacitor device testing as shown by Demarconnay et. al. and Malak-Polaczyk [25, 26]. The material to be studied was sandwiched between these two electrodes, with the PVDF membrane acting as a separator. The electrolyte used was $1 \mathrm{M} \mathrm{H}_{2} \mathrm{SO}_{4}$ that was inserted through the top nut. For the stacked cell configuration, each layer of the 90\% SWNT $10 \%$ mw rGO were each stacked in between stainless steel mesh, with each electrode comprising of five layers. Material loading was dependent on the film thickness of the active electrode material with $1 \mu \mathrm{m}$ equalling $0.108 \mathrm{mg} / \mathrm{cm}^{2}$ up to 4.91 $\mathrm{mg} / \mathrm{cm}^{2}$ for the $21 \mu \mathrm{m}$ thickness. Thickness was optimised for device testing at $17 \mu \mathrm{m}$ with a mass loading of $3.46 \mathrm{mg} / \mathrm{cm}^{2}$ for each electrode. Therefore the SEC had 5 layers, with the total mass of active material equalling $17.3 \mathrm{mg} / \mathrm{cm}^{2}$. Guidelines were followed from Gogotsi et. al. [27]. The CV response of the 
electrodes was measured using an EDAQ Australia system with EChem V2 software (ADI Instruments Pty Ltd.) with a potential difference of $1.1 \mathrm{~V}$. All EIS measurements were performed at room temperature where the frequency range spanned $100 \mathrm{kHz}$ to $0.1 \mathrm{~Hz}$ with an AC amplitude of $10 \mathrm{mV}$ (rms) at OCP using a Gamry EIS 3000TM system. For longterm cyclability of the device, Galvanostatic cycling tests were carried out with a Neware potentiostat, Test Control V.5.0 software, able to record a point every $1 \mathrm{~s}$. The potential window studied was between $0 \mathrm{~V}$ and $1.1 \mathrm{~V}$. In all device tests, the electrolyte used was aqueous $1 \mathrm{M} \mathrm{H}_{2} \mathrm{SO}_{4}$ due to its good conductivity and compatibility with carbon surfaces [28]. Series resistance modelling was performed using ZView V 3.2, Scribner Associates. All plasma treatments were performed directly before device assembly to ensure the treatment remains stable.

\section{Physical characterization.}

Physical characterisation was achieved by scanning electron microscopy (SEM), X-ray photoelectron spectroscopy (XPS), X-ray diffraction (XRD) and Brunauer, Emmett, Teller (BET) surface area and Raman spectroscopy. SEM was able to show the morphology of the SWNT- mw rGO composite. Raman spectroscopy was used to assess the vibrational properties of the hybrid material. SEM images were obtained from a JEOL JSM-7500FA field emission SEM. For SEM the accelerating voltage was $2.0 \mathrm{kV}$ with the emission current being set at 10 $\mu \mathrm{A}$. Raman spectroscopy was carried out on a Jobin-Yvon Horbia 800 using a $633 \mathrm{~nm}$ laser. The data analysis was carried

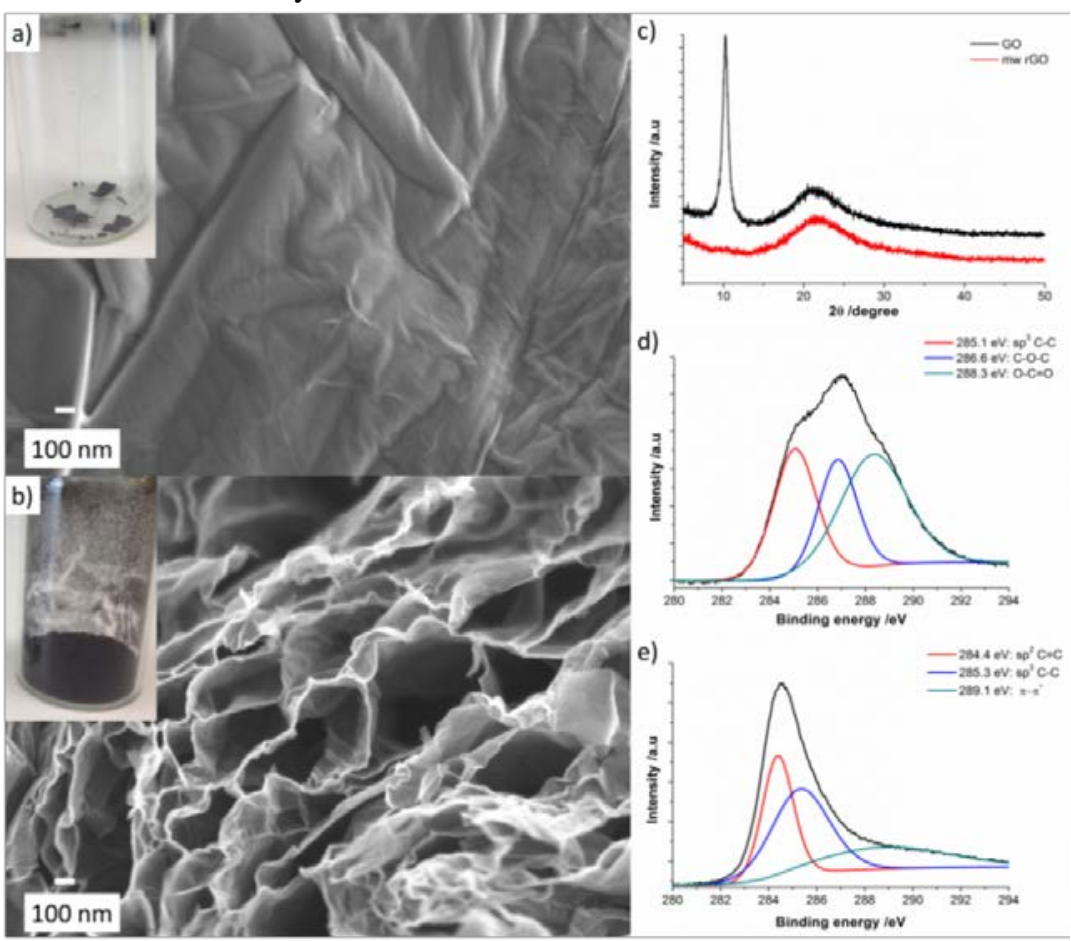

Figure 1: SEM image (with inset) of GO and mw rGO. (a) Before \& (b) After microwave irradiation. (c) XRD spectra. (d) \& (e) Comparison of the XPS C1s spectra. out using Labspec V.5.45.09 software. X-ray photoelectron spectroscopy (PHOIBOS 100 hemispherical energy analyser from SPECS) was done using $\mathrm{Al} \mathrm{K}_{\alpha}$ radiation $(1486.6 \mathrm{eV}$ ) in fixed analyser transmission mode. X-ray diffraction (XRD) was performed on a GBC MMA XRD $(\lambda=1.54 \AA)$ with the voltage and current kept at $-40 \mathrm{kV}$ and $25 \mathrm{~mA}$ respectively. BET measurements were performed using an Autosorb IQ gas adsorption system from Quantachrome Instruments тм with ASiQWin V.1.11 software.

\section{Results \& Discussion}

The SEM images of Figure 1a and Figure 1b are of the raw GO and mw rGO powders. From Figure 1, some qualitative observations can be made. It can be seen that the GO structure is very uniform, containing valleys and elevated regions, which reflect vast amounts of sheet stacking. In Figure 1b, after microwave irradiation; the GO expands leading to the development of an accordion type structure that is highly porous, forming an interconnected network with minimal restacking [24].

The increased porosity and thus surface area (observed from electrochemical testing) could play a key role in the development and preparation of composite electrode materials. Optical image comparisons (before and after microwave irradiation) are shown in the insets of Figure 1a and Figure 1b where the abrupt change in volume expansion is clearly visible. 


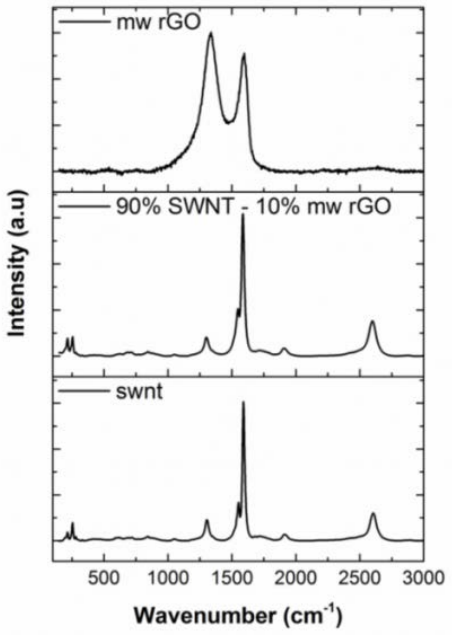

Figure 2: Raman spectroscopy of mw rGO, 90\% SWNT-10\% mw rGO, and SWNT electrodes after 20 minutes plasma treatment. Laser line used was $632.81 \mathrm{~nm}$.

Microwave irradiation of the GO led to exfoliation through the removal of volatile species from the interlayer spaces of the graphene intercalation compound [23, 24]. The XRD spectra of Figure 1c shows the two distinct peaks for GO at $2 \theta=10.2 \mathrm{o}$ and $22.0 \mathrm{o}$, and one distinct peak for mw rGO at $2 \theta=22^{\circ}$. The sharp peak at $10.2^{\circ}$ is characteristic of GO powder that corresponds to an interlayer distance of $0.87 \mathrm{~nm}$ [29, 30]. The broader peak at $2 \theta=22^{\circ}$ corresponds to the (002) crystal plane of graphite and amorphous carbon [31]. It is clear that after exfoliation using microwave irradiation, the sharp peak at $2 \theta=$ $10.2^{\circ}$ is suppressed as a result of the rapid expansion of the GO layers. The broadness of the XRD peak from all samples is most likely due to either an increase in disorder of the throughplane direction and/or structural defects inducded by probe sonication [29]. The (002) plane for $100 \% \mathrm{mw}$ rGO is slightly shifted due to the smaller interplanar spacing of the GO sheets (4.04 Å compared to $4.43 \AA$ ).

The Raman spectra observed in Figure 2 depicts mw rGO, 90\% SWNT - 10\% mw rGO, and SWNT composite electrodes. The SWNT response is very much a characteristic of single walled nanotubes where the radial breathing modes are located between $150 \mathrm{~cm}^{-1}$ and $305 \mathrm{~cm}^{-1}$ representing the coherent vibration of the $C$ atoms in the radial direction [32]. The ratio of the $\mathrm{D} / \mathrm{G}$ band ( $\mathrm{D}$ band is the disorder induced band arising from defects in the walls of the nanotubes; the $G$ band is a result of phonon wave confinement along the circumferential direction of the nanotube that leads to tangential C-C stretching transitions) of 0.150 indicates a high degree of purity for the SWNTs [32, 33]. The shoulder peak $\left(1553 \mathrm{~cm}^{-1}\right)$ to the left of the $\mathrm{G}$ band $\left(1591 \mathrm{~cm}^{-1}\right.$ ) is most likely from the effect of functional groups on the $\mathrm{sp}^{2}$ breathing mode [32]. As the ratio of SWNT is decreased (and subsequently mw rGO is increased), it can be seen that the $\mathrm{D}$ band of the composite becomes broader. This broadening is thought to be two D band peaks associated with SWNT and mw rGO with the D band of the mw rGO becoming stronger as its weight ratio is increased. When considering mw rGO only, the Raman spectra is characteristic of a $\mathrm{sp}^{2}$ hybridised material that contains defects on the graphene / graphite basal planes.

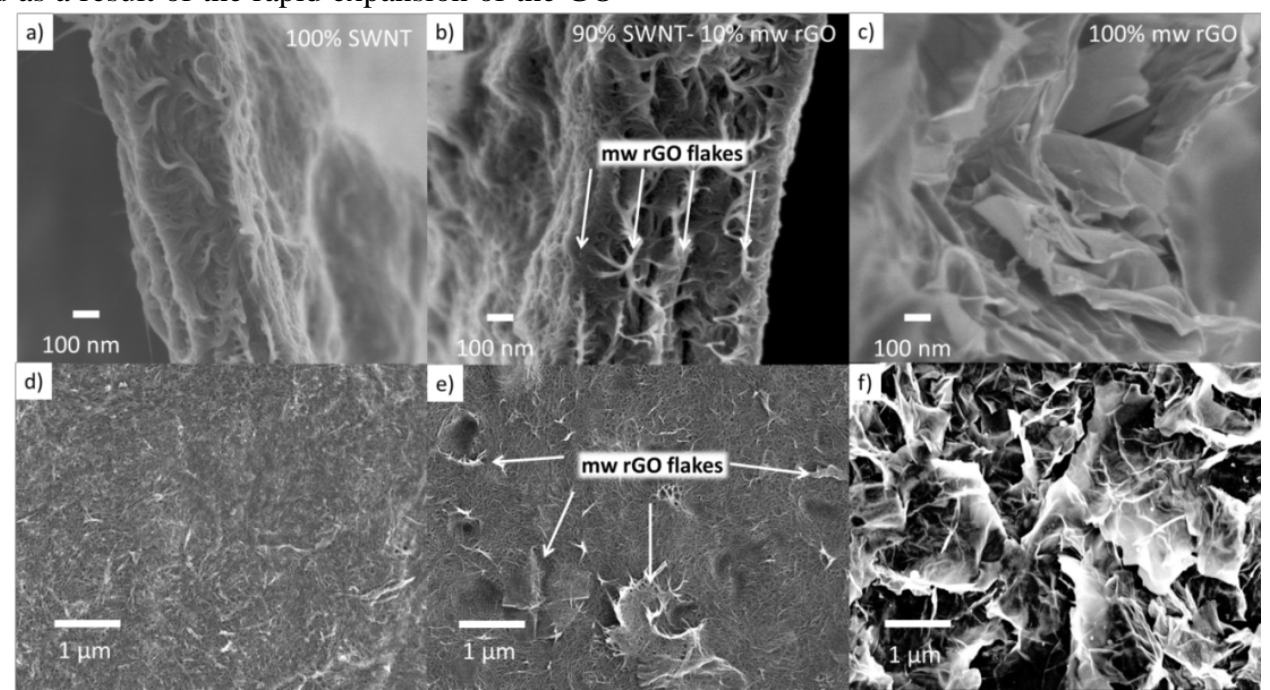

Figure 3: SEM images of SWNT - mw rGO composite with varying weight ratios. Top row is cross-sectional area, bottom row is surface image (a) \& (d) 100\% SWNT, (b) \& (e) $90 \%$ SWNT- $10 \%$ mw rGO, (c) \& (d) $100 \%$ mw rGO.

The D band peak of GO occurs at $1328 \mathrm{~cm}^{-1}$; while the $\mathrm{G}$ band peak is observed at $1586 \mathrm{~cm}^{-1}$ [34]. An extremely weak 2D peak is present at $2616 \mathrm{~cm}^{-1}$ which is characteristic of chemically converted graphene oxide as full conversion to pure 
graphene does not occur [34]. The Raman spectra of all composites can be seen in the supporting section, Table S1 and Figure S1.

In Figure 3 cross-section and surface images of SWNT, $90 \%$ SWNT - 10\% mw rGO, and $100 \%$ mw rGO composite electrodes are shown. When considering the 100\% SWNT film (Figure 3a and Figure 3d), the SWNTs are distributed in a nonuniform manner with varying bundle sizes and some agglomeration being apparent. Images of the the 90\% SWNT$10 \%$ mw rGO composite (Figure $3 \mathrm{~b}$ and Figure $3 \mathrm{e}$ ) reveal a layering effect due to $\pi-\pi$ stacking interactions wherein mw rGO sheets are covered with SWNTs. A highly porous structure is generated at the interface of these two nano-carbons. Furthermore, the mw rGO penetrates into the nanotube system, acting as anchor sites which improves electrode conductivity; as evidenced by reduction of the series resistance to $4 \mathrm{Ohm} . \mathrm{cm}^{2}$ of the $90 \%$ SWNT-10\%mw rGO film (Figure S3) [35]. It is most likely that the high surface energy of the mw rGO edges attracts the ends of the SWNTs and forms a connection. The surface image shows mw rGO islands / platelets that are uniformly dispersed throughout the SWNT matrix. tThis effect is thought to better tailor the micro-porosity and meso-porosity of the system which maximises the available surface area. The $100 \%$ mw rGO film (Figure 3c and Figure 3f) contains a random distribution of sheets that are crumpled in nature with some restacking effects apparent.

The BET surface areas of the mw rGO and SWNTs powders have been measured at $893 \mathrm{~m}^{2} / \mathrm{g}$ and $1000 \mathrm{~m}^{2} / \mathrm{g}$ respectively. When considering electrode performance, it is important to take into account the ESA. A comparison of the ESA for all the composites (Table S2) reveals that the $90 \%$ SWNT-10\% mw rGO films has the largest ESA $\left(52.9 \mathrm{~cm}^{2}\right)$

Plasma treatment of the electrode materials prior to electrochemical testing was done in order to improve the wettability of the electrode surface (without changing the properties of the bulk) through the addition of functional groups [36]. This is achieved through thermal oxygen atoms interacting chemically with the surface atoms leading to the formation of oxygen-rich functional groups [36]. Contact angle measurements of the $90 \%$ SWNT-10\%mw rGO composite electrodes (Figure S4a and Figure S4b) prior to $\left(54.5^{\circ}\right.$ ), and after plasma treatment $\left(<1^{\circ}\right)$ indicate an enhancement in the wettability of the electrodes towards water [37].

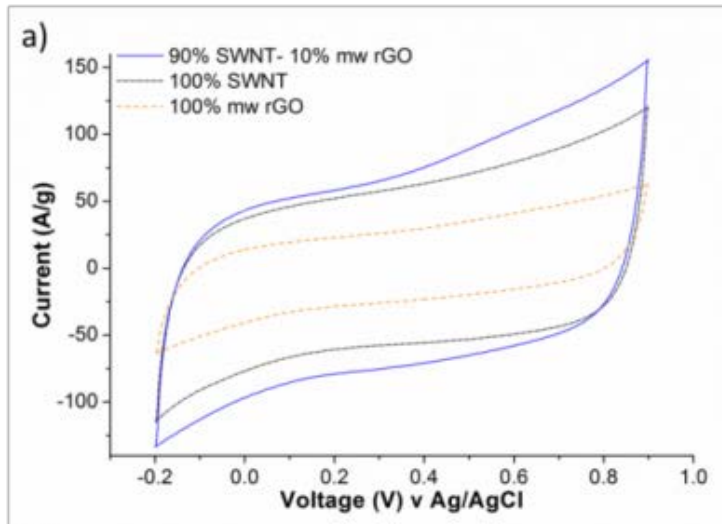

b)
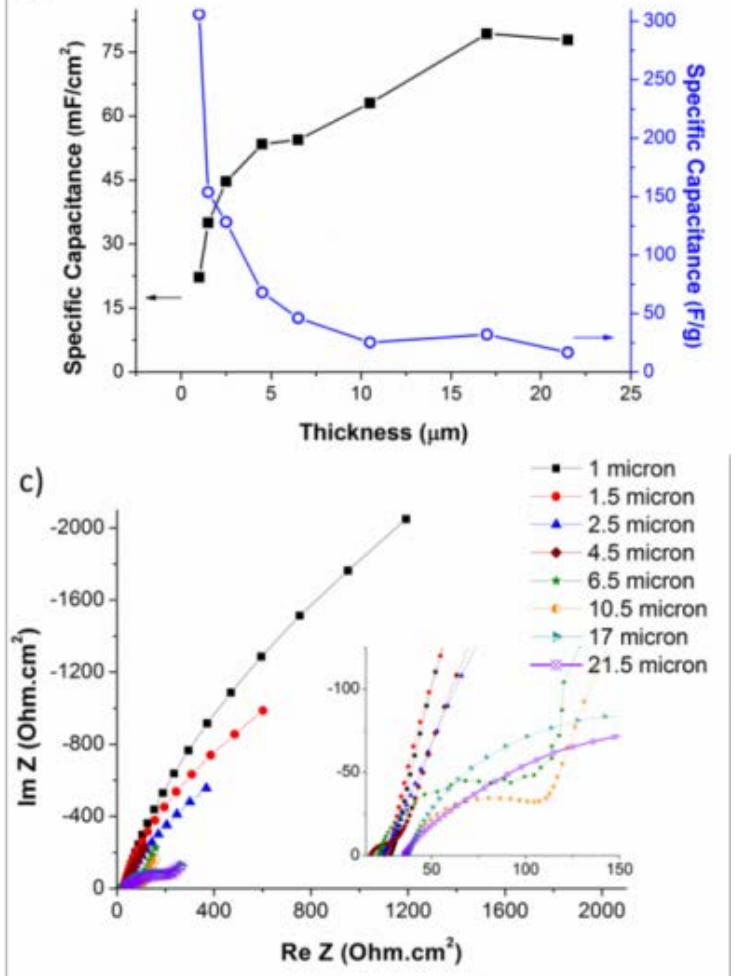

Figure 4: SWNT : mw rGO composite electrode. (a) Cyclic voltammetry at $500 \mathrm{mV} / \mathrm{s}$ of $100-0,90-10$ and $0-100$ (b) Specific capacitance calculated at $20 \mathrm{mV} / \mathrm{s}$ as the thickness is increased. (c) Nyquist plot of $90 \%$ SWNT-10\% mw rGO electrodes with varied thicknesses from 1 micron to 21.5 micron. System is three electrode with a Pt mesh counter (2 $\mathrm{cm} 2$ ) and $1 \mathrm{M} \mathrm{NaNO}_{3} / \mathrm{H}_{2} \mathrm{O}$.

The CVs observed in Figure 4a display a highly rectangular nature indicative of electric double layer capacitance at a scan rate $500 \mathrm{mV} / \mathrm{s}$ [13]. The 90\% SWNT-10\%mw rGO film achieved the highest specific capacitance of $120 \mathrm{~F} / \mathrm{g}$ at a scan rate of $500 \mathrm{mV} / \mathrm{s}$, and $306 \mathrm{~F} / \mathrm{g}$ at $20 \mathrm{mV} / \mathrm{s}$. A full $\mathrm{CV}$ comparison of all composites reveals the largest electroactive surface area is obtained by the $90 \%$ SWNT-10\%mw rGO film (supporting section, Figure S2). In Figure 4b, films of $90 \%$ SWNT - 10\% mw rGO were made with thickness in order to establish the maximum amount of utilisable surface area. The 
specific capacitance was calculated at $20 \mathrm{mV} / \mathrm{s}$ and plotted against electrode thickness as is shown in Figure $4 \mathrm{~b}$.

As the thickness of the film increases from $1 \mu \mathrm{m}$ to $21.5 \mu \mathrm{m}$ (Figure 4b), the capacitive current per unit area increases as there is more material that interacts with the electrolyte. However, the capacitive current per unit mass decreases as not all of the electroactive surface area is being utilized. The limiting current per unit area is for the 17 micron as it plateaus off after this point. The effects of an increase in thickness can be seen in the Nyquist plot (Figure 4c) wherein the width of the high frequency semi-circle that corresponds to the polarisation resistance $\left(R_{p}\right)$ increases with thickness. The inset of Figure 4c shows that $R_{p}$ is 3.4 ohm.cm ${ }^{2}$ for the $1 \mu \mathrm{m}$ film and scales to $117.5 \mathrm{Ohm} \mathrm{cm}^{2}$ for the $21.5 \mu \mathrm{m}$ film. $\mathrm{R}_{\mathrm{p}}$ rises with film thickness due to an increase in diffusion length, as well as a change in the porosity of the electrode. These factors have an adverse effects on the rate of electrolyte diffusion; i.e. it becomes more sluggish [38]. Furthermore, for the 10.5, 17, and $21.5 \mu \mathrm{m}$ thicknesses, the series resistance $\left(\mathrm{R}_{\mathrm{s}}\right)$ slightly shifts to
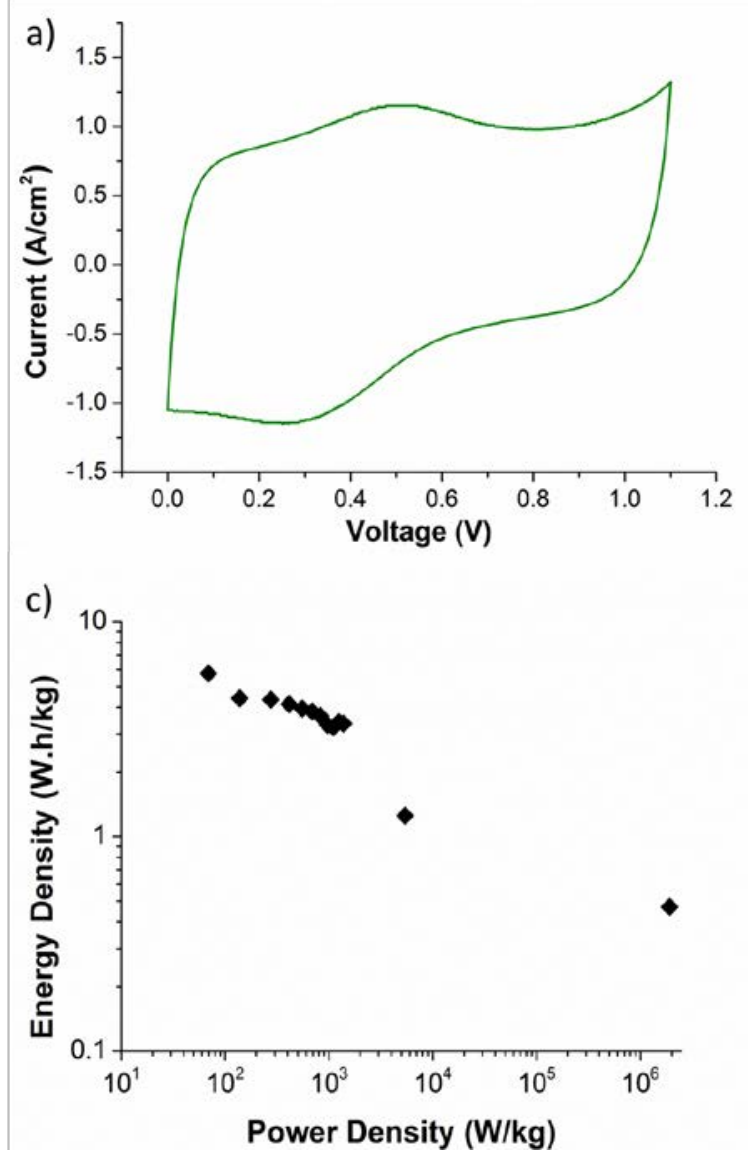

the right; i.e. $R_{s}$ is greater for the thicker films. Coupled to this in the middle frequency portion of the spectrum, there is a slight extenuation of the Warburg diffusion region which also indicates sluggish diffusion [39, 40]. Specific capacitance (F/g) calculations can be found in the supporting information.

Supercapacitor device fabrication was carried out for the $90 \%$ SWNT-10\% mw rGO electrode with a thickness of 17 microns using a Swagelok test cell as can be seen from Figure 5. Figure 5a, the $\mathrm{CV}$ of $90 \% \mathrm{SWNT}-10 \% \mathrm{mw}$ rGO in $\mathrm{H}_{2} \mathrm{SO}_{4}$ shows a rectangular behavior due to the electric double layer capacitance, while the reversible redox peaks at $0.3 \mathrm{~V}$ and 0.55 $\mathrm{V}$ are due to pseudocapacitance arising from the interaction of functional groups of the composite material and the sulphuric acid [28, 41]. Sulphuric acid enhances the redox behaviour as it is thought that $\mathrm{H}+$ or $\mathrm{OH}^{-}$must be involved in the electrochemical reactions according to the strong dependence of pseudocapacitance on the $\mathrm{H}+$ concentration [28] (see supporting Figure S5).

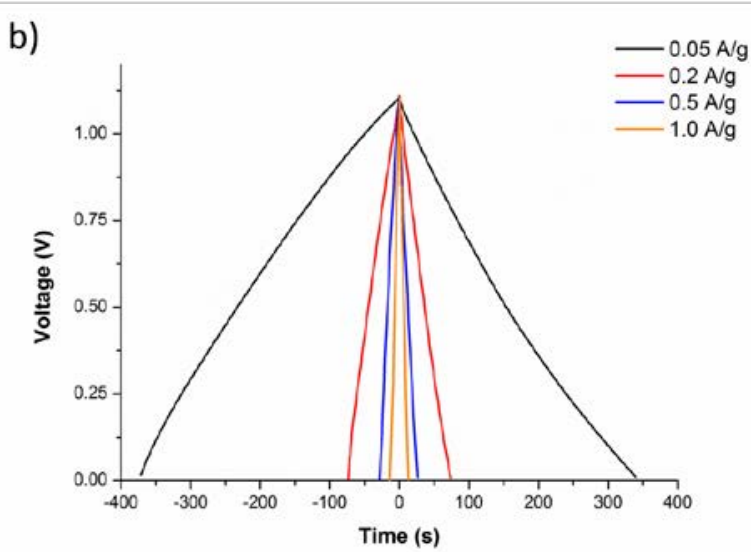

d)

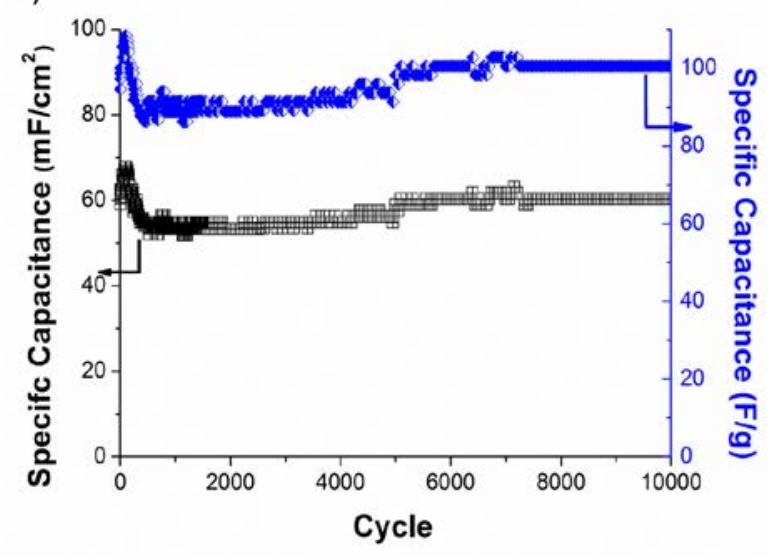

Figure 5: (a) $\mathrm{CV}$ at $20 \mathrm{mV} / \mathrm{s}\left(1 \mathrm{M} \mathrm{H}_{2} \mathrm{SO}_{4} / \mathrm{H}_{2} \mathrm{O}\right)$ (b) Galvanostatic charge / discharge curves for different current loadings. (c) Ragone plot of power density versus energy density. (d) $\mathrm{C}_{\mathrm{sp}}$ versus cycle number for varied current loadings at $1.0 \mathrm{~A} / \mathrm{g}$.

In Figure 5b, galvanostatic charge / discharge (GCD) curves show a symmetrical behaviour, but with a slight curvature at low current rates of $0.1 \mathrm{~A} / \mathrm{g}$ and $0.2 \mathrm{~A} / \mathrm{g}$ indicating pseudocapacitance[27]. In the Ragone plot of Figure 5c, it can be seen that the $90 \%$ SWNT - 10\% mw rGO displays a high energy density of $6 \mathrm{Wh} / \mathrm{kg}$ and a maximum power density 1.9 $\mathrm{MW} / \mathrm{kg}$ respectively.

To compare these single electrode results with commercially available supercapacitors, Murata polymer and electric double layer capacitors range from $10 \mu \mathrm{F}-470 \mathrm{mF} 38$. Generally speaking the energy and power density range can 
range from $0.1-10 \mathrm{Wh} / \mathrm{kg}$ to $1-100 \mathrm{~kW} / \mathrm{kg} 18$, 39. Long term stability testing (Figure $5 \mathrm{~d}$ ) over 10000 cycles at $1.0 \mathrm{~A} / \mathrm{g}$ shows and extremely stable and reversible response of the electrode during the charge / discharge process, with the capacity retention of $95 \%$ at cycle 10 000. The initial increase in capacitance is due to an initial activation where full wetting and volume expansion / contraction equilibrates the system [40].

A SEC has been constructed by separating five layers of the 90\% SWNT-10\% mw rGO with a stainless steel current collector on each electrode as shown in the schematic diagram (Figure 6b). The design in this manner enhances the ability of the electrolyte ions to access the active surface area, thus improving the ESA and increasing the energy density. The device performance (per electrode volume and excluding the current collector) is increased as this is important to commercial standards and applications [1].

To demonstrate the overall performance of the SEC, the current rate has been switched over 1500 cycles. In Figure 6c, the current rate has been switched from $0.1 \mathrm{~A} / \mathrm{g}$ (cycle 1-250), up to $10 \mathrm{~A} / \mathrm{g}$ (cycle 1001-1250) and back to $0.1 \mathrm{~A} / \mathrm{g}$ (cycle 1251-1500) with the SEC showing a reversible behaviour as the capacity retention at $1.0 \mathrm{~A} / \mathrm{g}$ was $97 \%$.
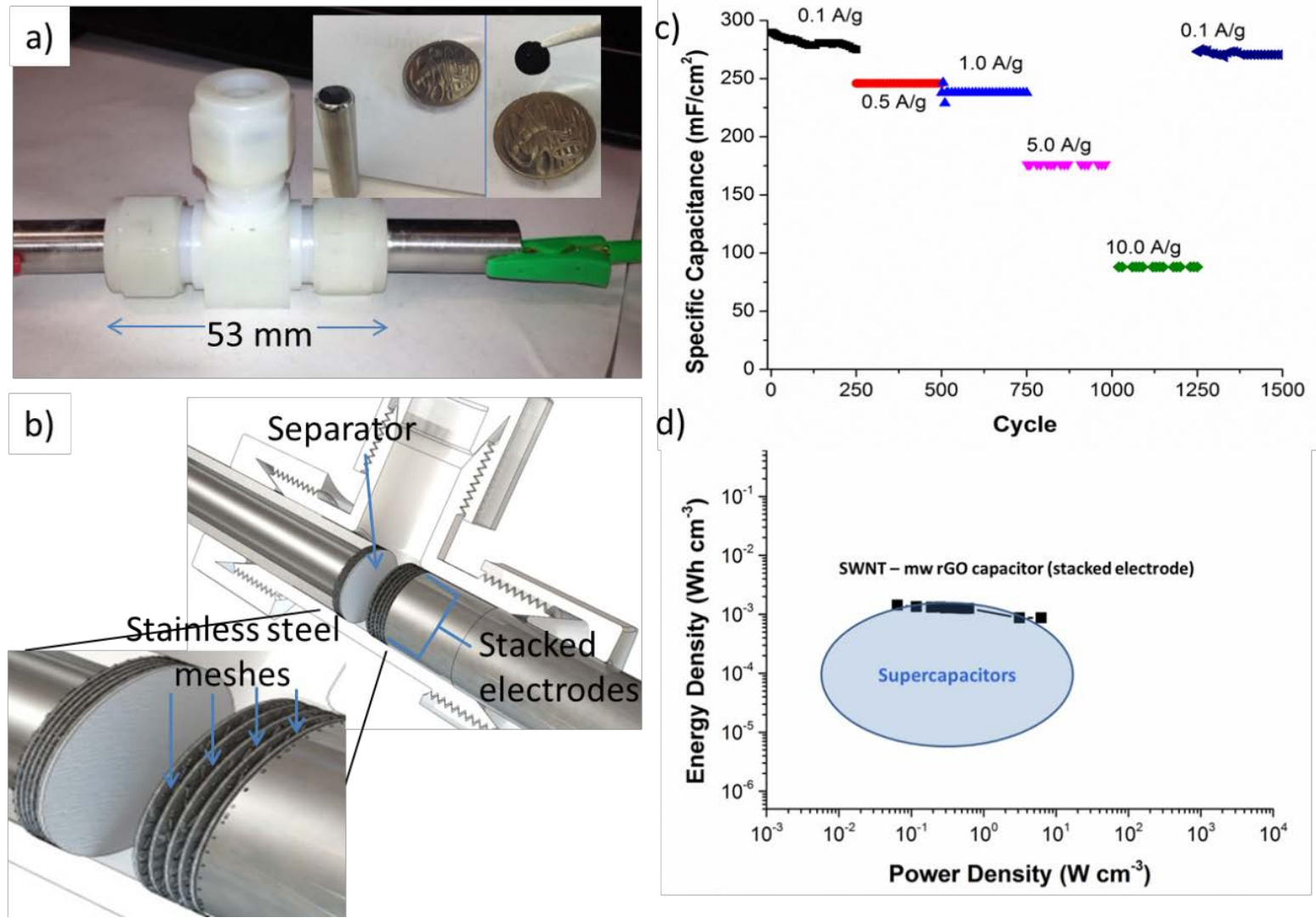

Figure 6: (a) Optical image of swagelok test cell (b) Rendered image with zoomed in region showing the stacked electrodes separated by stainless steel mesh. (c) $\mathrm{C}_{\mathrm{sp}}$ versus cycle number for varied current loadings. (d) Comparison of energy and power densities of the SEC with current supercapacitor research [42]'[43].

The volumetric energy density is $1.43 \mathrm{~mW} \cdot \mathrm{hr} / \mathrm{cm}^{3}$ and the power density is $6.25 \mathrm{~W} / \mathrm{cm}^{3}$ respectively as is shown by the Ragone plot of Figure 6d. Here, there is little decrease in the energy density indicating that this configuration has stable energy output over the given power range. Comparatively, our $90 \%$ SWNT-10\% mw rGO device is on the upper region of current supercapacitor work (see Figure 6d, Ragone plot).

Long term stability testing of the stacked electrode device at 1.0 A/g occurred for 10000 cycles as shown in Figure 7a. The initial capacitance is $271 \mathrm{mF} / \mathrm{cm}^{2}$ while at cycle 10000 the capacitance is $251 \mathrm{mF} / \mathrm{cm}^{2}$ indicating a capacity retention of 93\%. When comparing the Nyquist plots of Figure 7b, stability is indicated once more as there is no change in the Nyquist plot from cycle 5000 to 10000 as the $R_{p}$ is identical (15.7 Ohm.cm²) The initial Nyquist plot contains a smaller $R_{p} 5.8$ Ohm. $\mathrm{cm}^{2}$ due to the fact that the all of the active material of the electrode has not been fully wetted, electrolyte ions have not sufficiently penetrated into the composite, and there has been no significant volume change[44]. The SEC configuration had approximately a fourfold increase in performance $\left(250 \mathrm{mF} / \mathrm{cm}^{2}\right)$ as compared to the single electrode configuration $\left(60 \mathrm{mF} / \mathrm{cm}^{2}\right)$. From the SEM image of Figure 7c and Figure 7d, it can be seen that there is no observable difference in the physical structure of the $90 \%$ SWNT - 10\% mw rGO electrode before and after 10000 cycles of GCD. In both cases mw rGO is covered and separated by SWNTs. However, it is apparent that after GCD in Figure 7c 
and Figure $7 d$, the electrode is denser due to the cell configuration that presses both electrodes and separator between the stainless steel plates. Overall the electrode is stable and robust thus maintaining its integrity as it undergoes the charge / discharge process which is extremely important in practical applications where consistency and reliability must be
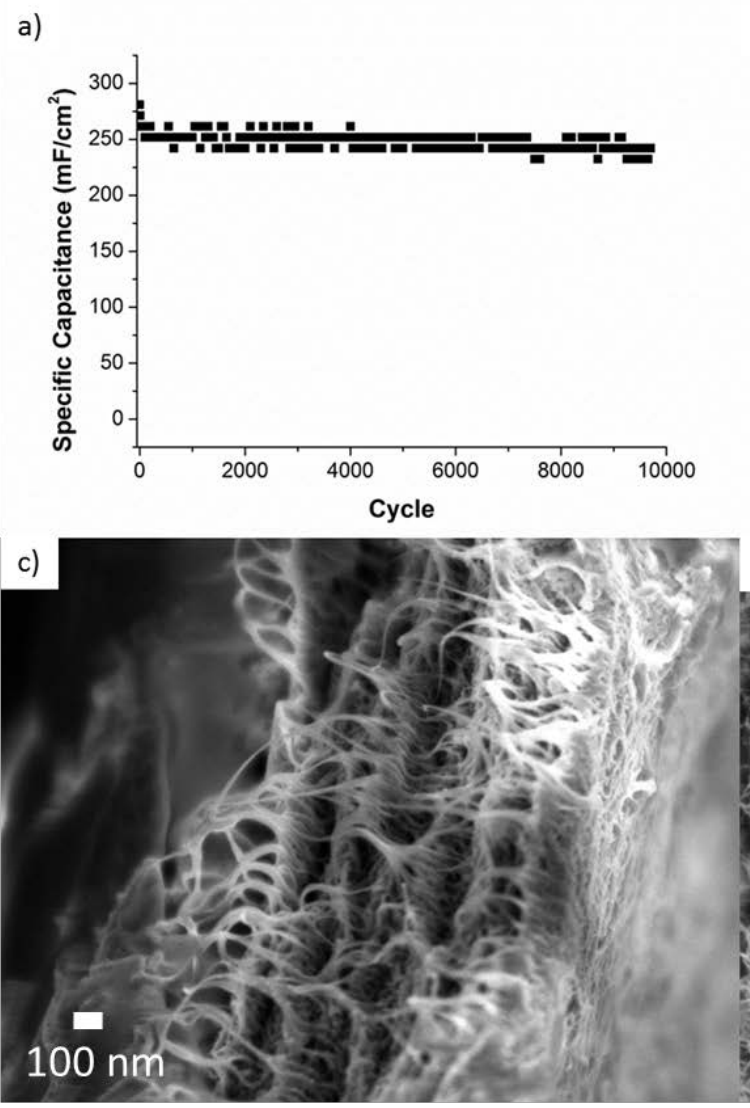

guaranteed. Equations for capacitance (C), energy density $(E)$, power density $(P)$ and maximum power density $\left(P_{\max }\right)$ as either a function of mass or unit volume can be found in the supporting information section [38, 45]:

b)
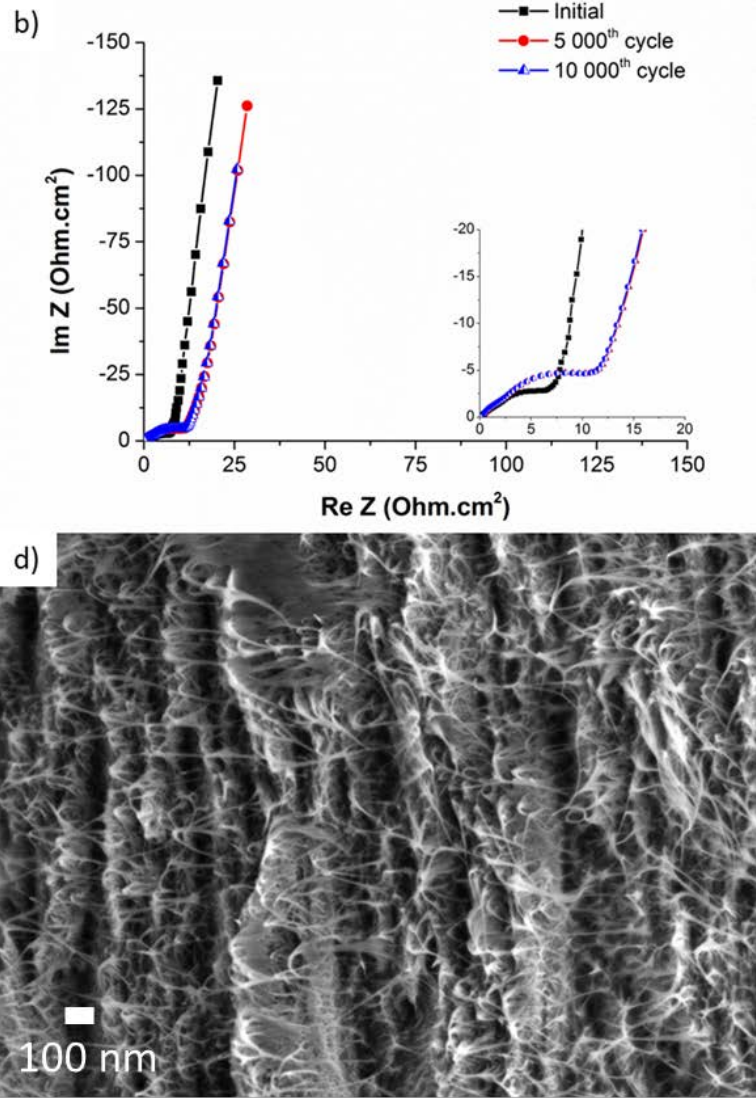

Figure 7: (a) Long term cyclability at $1.0 \mathrm{~A} / \mathrm{g}$ using GCD. (b) Nyquist plot prior to and after 5000 and 10000 cycles. SEM images of 1 layer from the stacked electrode configuration before (c) and after (d) 10000 cycles of GCD.

\section{Conclusions}

In summary, a composite material comprised of SWNTs and mw rGO has been successfully synthesised and fabricated into a composite electrode material. The composite was optimised as an electrode material for use in supercapacitors. SEM showed a structure wherein sheets of mw rGO were intertwined in a SWNT matrix, thus tailoring the micro and meso-porosity which maximises the ESA.

Raman spectroscopy showed SWNT to have a characteristic response, while the mw rGO showed a large D/G band ratio. The $\mathrm{D}$ band peak of the composite also broadened as the weight ratio of mw rGO increased due to the large $\mathrm{D} / \mathrm{G}$ ratio of pure mw rGO. The optimum ratio for the electrode which maximises capacitance was calculated to be $90 \%$ SWNT - 10\% mw rGO with 20 minutes plasma treatment time in order to maximise the wettability of the electrode surface (capacitance $306 \mathrm{~F} / \mathrm{g}$ ). It was found that a thickness of 17 micron was optimum in order to ensure that the capacitance per unit area was maximised.
Long term testing of the device showed excellent stability over 10000 cycles with $95 \%$ capacity retention. EIS over the course of long term testing showed little change indicating a stable and robust electrode.

A SEC with 5 layers per electrode was employed in order to show that our material is suitable for real world applications where volumetric density is important. The Ragone plot displays a performance that is up at the top range of supercapacitor device performance per total volume. GCD displayed high reversibility up to $10 \mathrm{~A} / \mathrm{g}$ and long term stability over 10000 cycles with 93\% capacity retention. The Nyquist plots showed little change while the SEM images of the composite structure showed no visual changes. This SEC showed a 4 fold increase in the specific capacitance as compared to the single electrode device

\section{Acknowledgements}

The authors acknowledge the financial support from the Australian Research Council Discovery (DP0877348) and 
Linkage Programs (LP0989266), CAP-XX Australia Pty. Ltd.. They acknowledge the UoW Electron Microscopy Centre and Australian National Fabrication Facility for provision of services and equipment access.

\section{Notes and references}

Electronic Supplementary Information (ESI) available: [details of any supplementary information available should be included here]. See DOI: 10.1039/b000000x/

1. Niu, Z., et al., Compact-designed supercapacitors using free-standing single-walled carbon nanotube films. Energy and Environmental Science, 2011. 4(4): p. 1440-1446.

2. Presser, V., M. Heon, and Y. Gogotsi, Carbide-Derived Carbons From Porous Networks to Nanotubes and Graphene. Advanced Functional Materials, 2011. 21(5): p. 810-833.

3. Zhang, L.L. and X.S. Zhao, Carbon-based materials as supercapacitor electrodes. Chemical Society Reviews, 2009. 38(9): p. 2520-2531.

4. Woo, S., et al., Synthesis of a graphene - carbon nanotube composite and its electrochemical sensing of hydrogen peroxide. Electrochimica Acta, 2012. 59(1): p. 509-514.

5. Buglione, L. and M. Pumera, Graphene/carbon nanotube composites not exhibiting synergic effect for supercapacitors: The resulting capacitance being average of capacitance of individual components. Electrochemistry Communications, 2012. 17(1): p. 45-47.

6. Cheng, Q., et al., Graphene and carbon nanotube composite electrodes for supercapacitors with ultra-high energy density. Physical Chemistry Chemical Physics, 2011. 13(39): p. 17615-17624.

7. Jae Young Kim, et al., Graphene-carbon nanotube composite as an effective conducting scaffold to enhance the photoelectrochemical water oxidation activity of a hematite film. The Royal Society of Chemistry, 2012.

8. Y. Lan, Y. Wang, and Z.F. Ren, Physics and applications of aligned carbon nanotubes. Advances in Physics, 2011. 60(4): p. 553-678.

9. Zilli, D., et al., Formation mechanism of Y-junctions in arrays of multi-walled carbon nanotubes. Colloids and Surfaces A: Physicochemical and Engineering Aspects, 2008. 327(1-3): p. 140143.

10. Meyyappan, M., Carbon Nanotubes: Science and Applications. 2005, Boca Raton: CRC Press.

11. Lota, G., K. Fic, and E. Frackowiak, Carbon nanotubes and their composites in electrochemical applications. Energy and Environmental Science, 2011. 4(5): p. 1592-1605.

12. Novoselov, K.S., et al., A roadmap for graphene. Nature, 2012. 490(7419): p. 192-200.

13. Bose, S., et al., Carbon-based nanostructured materials and their composites as supercapacitor electrodes. Journal of Materials Chemistry, 2012. 22(3): p. 767-784.

14. Byrne, M.T. and Y.K. Gun'ko, Recent Advances in Research on Carbon Nanotube-Polymer Composites. Advanced Materials, 2010. 22(15): p. 1672-1688.

15. Centi, G. and S. Perathoner, Carbon Nanotubes for Sustainable Energy Applications. ChemSusChem, 2011. 4(7): p. 913-925.

16. Jalili, R., et al., Organic Solvent-Based Graphene Oxide Liquid Crystals: A Facile Route toward the Next Generation of Self-
Assembled Layer-by-Layer Multifunctional 3D Architectures. ACS Nano, 2013. 7(5): p. 3981-3990.

17. Romano, M.S., et al., Carbon Nanotube - Reduced Graphene Oxide Composites for Thermal Energy Harvesting Applications. Advanced Materials, 2013: p. n/a-n/a.

18. Li, Q., et al., Structural evolution of multi-walled carbon nanotube/MnO2 composites as supercapacitor electrodes. Electrochimica Acta, 2011. 59(0): p. 548-557.

19. Aboutalebi, S.H., et al., High-Performance Multifunctional Graphene Yarns: Toward Wearable All-Carbon Energy Storage Textiles. ACS Nano, 2014. 8(3): p. 2456-2466.

20. El-Kady, M.F. and R.B. Kaner, Scalable fabrication of high-power graphene micro-supercapacitors for flexible and on-chip energy storage. Nat Commun, 2013. 4: p. 1475.

21. Marcano, D.C., et al., Improved Synthesis of Graphene Oxide. ACS Nano, 2010. 4(8): p. 4806-4814.

22. Hirata., US Patent 6596 396. 2003.

23. Falcao, E.H.L., et al., Microwave exfoliation of a graphite intercalation compound. Carbon, 2007. 45(6): p. 1367-1369.

24. Tryba, B., A.W. Morawski, and M. Inagaki, Preparation of exfoliated graphite by microwave irradiation. Carbon, 2005. 43(11): p. 24172419.

25. Demarconnay, L., E. Raymundo-Pinero, and F. Béguin, A symmetric carbon/carbon supercapacitor operating at $1.6 \mathrm{~V}$ by using a neutral aqueous solution. Electrochemistry Communications, 2010. 12(10): p. $1275-1278$.

26. Malak-Polaczyk, A., C. Vix-Guterl, and E. Frackowiak, Carbon/Layered Double Hydroxide (LDH) Composites for Supercapacitor Application Energy \& Fuels, 2010. 24(6): p. 33463351.

27. Gogotsi, Y. and P. Simon, True performance metrics in electrochemical energy storage. Science, 2011. 334(6058): p. 917918.

28. Chen, Q.-L., et al., Fabrication and electrochemical properties of carbon nanotube array electrode for supercapacitors. Electrochimica Acta, 2004. 49(24): p. 4157-4161.

29. Zhu, Y., et al., Exfoliation of Graphite Oxide in Propylene Carbonate and Thermal Reduction of the Resulting Graphene Oxide Platelets. ACS Nano, 2010. 4(2): p. 1227-1233.

30. Li, Z., et al., Synthesis of hydrothermally reduced graphene/MnO2 composites and their electrochemical properties as supercapacitors. Journal of Power Sources, 2011. 196(19): p. 8160-8165.

31. Chen, Y., et al., One-pot synthesis of MnO2/graphene/carbon nanotube hybrid by chemical method. Carbon, 2011. 49(13): p. 44344442.

32. Dresselhaus, M.S., et al., Raman spectroscopy of carbon nanotubes. Physics Reports, 2005. 409(2): p. 47-99.

33. Salzmann, C.G., et al., The Role of Carboxylated Carbonaceous Fragments in the Functionalization and Spectroscopy of a SingleWalled Carbon-Nanotube Material. Advanced Materials, 2007. 19(6): p. 883-887.

34. Wu, Z.-S., et al., Doped Graphene Sheets as Anode Materials with Superhigh Rate and Large Capacity for Lithium Ion Batteries. ACS Nano, 2011: p. 5463-5471. 
35. Lee, S.H., et al., Tailored Assembly of Carbon Nanotubes and Graphene. Advanced Functional Materials, 2011. 21(8): p. 13381354.

36. Cvelbar, U., et al., Formation of functional groups on graphite during oxygen plasma treatment. Applied Surface Science, 2006. 253(4): p. 1861-1865.

37. Chau, T.T., et al., A review of factors that affect contact angle and implications for flotation practice. Advances in Colloid and Interface Science, 2009. 150(2): p. 106-115.

38. Taberna, P.L., P. Simon, and J.F. Fauvarque, Electrochemical Characteristics and Impedance Spectroscopy Studies of CarbonCarbon Supercapacitors. Journal of The Electrochemical Society, 2003. 150(3): p. A292-A300.

39. Wang, G., L. Zhang, and J. Zhang, A review of electrode materials for electrochemical supercapacitors. Chemical Society Reviews, 2012. 41(2): p. 797-828.

40. Naoi, K. and P. Simon, New Materials and New Configurations for Advanced Electrochemical Capacitors. Journal of The Electrochemical Society 2008. 17(1): p. 34-37.

41. Huang, Z.-D., et al., Effects of reduction process and carbon nanotube content on the supercapacitive performance of flexible graphene oxide papers. Carbon, 2012. 50(11): p. 4239-4251.

42. Pech, D., et al., Ultrahigh-power micrometre-sized supercapacitors based on onion-like carbon. Nat Nano, 2010. 5(9): p. 651-654.

43. Wu, Z.S., et al., Graphene-based in-plane micro-supercapacitors with high power and energy densities. Nat Commun, 2013. 4.

44. Sun, Y., Q. Wu, and G. Shi, Graphene based new energy materials. Energy \& Environmental Science, 2011. 4(4): p. 1113-1132.

45. H. Kurig, A. Janes, and E. Lust, Electrochemical Characteristics of Carbide-Derived Carbon|1-Ethyl-3-methylimidazolium Tetrafluoroborate Supercapacitor Cells. Journal of The Electrochemical Society, 2010. 157(3): p. A272-A279. 